\title{
Language in Alzheimer's Disease
}

\author{
Myron F. Weiner, M.D., Katherine E. Neubecker, B.A., Mary E. Bret, M.D., and Linda S. \\ Hynan, Ph.D. \\ University of Texas Southwestern Medical Center, Dallas (Drs. Weiner, Bret, and Hynan) and \\ University of Texas Health Science Center at San Antonio (Ms. Neubecker)
}

\begin{abstract}
Objective-To ascertain the clinical utility of language examination by psychiatrists in evaluating Alzheimer's disease (AD) patients.
\end{abstract}

Method-Data collected between 1986 and 2003 from a standardized psychiatric examination and neuropsychological testing of probable $\mathrm{AD}$ patients (diagnosed according to the criteria of the National Institute of Neurological and Communicative Disorders and Stroke and the Alzheimer's Disease and Related Disorders Association) were gathered from the database of the University of Texas Southwestern Alzheimer's Disease Center, Dallas. The variables studied were articulation, word-finding ability, hypofluency, hyperfluency, repetition, confrontational naming, and semantic (category) fluency Articulation, word-finding ability, hypofluency, hyperfluency, repetition, and confrontational naming were rated as normal or abnormal. Semantic fluency was scored numerically as the number of animals named in a minute. Cognitive impairment was assessed with the Mini-Mental State Examination (MMSE) and global impairment by the Clinical Dementia Rating (CDR) scale.

Results-There was a significant association $(\mathrm{p}<.0001)$ between MMSE and CDR scores for all language measures except hyperfluency. The MMSE scores were higher in the group with responses rated as normal compared to those with abnormal responses. Patients with greater cognitive and global impairment named fewer animals in a minute.

Conclusions-Abnormal articulation and repetition of words were unusual and therefore would not be useful for early detection, but when present, were associated with more advanced disease. Impairment in fluency, animal naming, and confrontational naming were common and increased in frequency with greater cognitive and global impairment. Because animal naming is a numerical measure, changes in the number of animals named in a minute can be used to monitor disease progression.

Evaluation of language can be an important part of the mental status examination, especially in the elderly, for whom the differential diagnosis includes dementing illnesses and in whom there is a need to quantify cognitive impairment. Nevertheless, there are few objective data concerning the relationship of language measures commonly used in the mental status examination to specific disease entities such as Alzheimer's disease (AD) and little information concerning the relationship between simple language measures and severity of cognitive impairment.

Corresponding author and reprints: Myron F. Weiner, M.D., University of Texas Southwestern Medical Center, 5323 Harry Hines Blvd., Dallas, TX 75390-9129, (myron.weiner@utsouthwestern.edu).

Poster presented at the annual meeting of the American Society for Geriatric Psychiatry, March 1-4, 2007, New Orleans, La.

The authors have no other personal affiliations or financial relationships with any commercial interest to disclose relative to the article. Disclosure of off-label usage: The authors have determined that, to the best of their knowledge, no investigational information about pharmaceutical agents that is outside U.S Food and Drug Administration-approved labeling has been presented in this article. 
It is generally agreed that subtle language deficits can be detected early in the course of AD based on complex linguistic scoring of picture-description tasks, ${ }^{1}$ but the mechanics of speech appear well preserved. Murdoch et a $1^{2}$ found that despite their difficulties with word finding and semantics, institutionalized AD patients did not exhibit greater impairment of articulation than the institutionalized control subjects. On the other hand, AD patients often have problems with fluency. Marczinski and Kertesz. ${ }^{3}$ compared the fluency of control subjects (mean Mini-Mental State Examination ${ }^{4}[\mathrm{MMSE}]$ score $=28.8$ ) with that of AD patients (mean MMSE score $=21.3$ ). Subjects performed 3 fluency tasks for 1 minute each, naming animals, grocery items, or words starting with the letter $S$ as rapidly as they could. The first 2 tasks tested semantic (categorical) fluency, and the last task tested phonemic fluency. The $\mathrm{AD}$ patients listed fewer items on all 3 tasks. In another study, ${ }_{5}^{5} \mathrm{AD}$ patients produced fewer animal names than controls and tended to list only farm animals, whereas control subjects provided several different clusters of animals. Cronin-Golomb et al. ${ }^{6}$ encountered similar results comparing 18 control subjects with $18 \mathrm{AD}$ patients with mild to severe dementia. For all 10 categories tested, AD patients listed fewer items than did control subjects. The $\mathrm{AD}$ patients produced a mean of only $50.6 \%$ of the number of items listed by control subjects, with individual categories ranging from $41 \%$ to $61 \%$. Moreover, within a given category, $\mathrm{AD}$ patients listed fewer subcategories and fewer items per subcategory. ${ }^{6} \mathrm{~A}$ more recent meta-analysis of 153 studies with nearly 16,000 participants indicated that semantic fluency is much more sensitive to the cognitive deficits in AD than is phonemic fluency. ${ }^{7}$

In another study, 8,918 institutionalized AD patients with a mean Mental Status Questionnaire ${ }^{10}$ score of 5.6 out of 10 were compared to 18 institutionalized, nonneurologically impaired control subjects who were matched for age, sex, and education. Relative to control subjects, the institutionalized AD patients committed a greater number of lexical errors when asked to name a target object. However, their word-finding difficulty did not appear to result from visual agnosia. The fact that incorrect answers often shared the same semantic category (such as fruit) as the correct answers and that AD patients could frequently describe the function of an object suggested that $\mathrm{AD}$ patients could still recognize the target object. ${ }^{8}$ When asked to describe a picture, $\mathrm{AD}$ patients could give as much information as control subjects, but they required more time and words to do so, indicating impaired grammatical semantic skills in AD patients. ${ }^{9}$

In this study, we investigated the relationship between simple language measures and degree of cognitive impairment in $\mathrm{AD}$, which we estimated using the MMSE, and the relationship of language measures to level of global impairment, as measured by the Clinical Dementia Rating ${ }^{11}$ (CDR) scale. The language measures examined by the investigators included articulation, fluency (word-finding ability, hypofluency, hyperfluency), semantic (category) fluency (ability to name animals), repetition (ability to repeat words and phrases), and confrontational naming.

\section{Method}

This study was based on information contained in the database of the University of Texas (UT) Southwestern Alzheimer's Disease Center, Dallas, collected between 1986 and 2003, during which time a standardized mental status examination, ${ }^{12}$ the MMSE, and the CDR were performed at the initial clinical visit. The language portion of the mental status examination included articulation, word-finding ability, hypofluency, hyperfluency, repetition, and animal naming. For those persons for whom animal naming was not done as part of the psychiatric examination, we used the score from the animal-naming portion of the Consortium to Establish a Registry for Alzheimer's Disease (CERAD) Neuropsychological Battery, ${ }^{13}$ which was also administered at the same time. We included data from the mental 
status examination of persons whose most current diagnosis was probable $\mathrm{AD}$ according to the criteria of the National Institute of Neurological and Communicative Disorders and Stroke and the Alzheimer's Disease and Related Disorders Association. ${ }^{14}$ The diagnosis of probable $\mathrm{AD}$ at initial clinical examination has a positive predictive value of 0.87 for autopsy-confirmed AD at the UT Southwestern Medical Center Alzheimer's Disease Center. ${ }^{15}$ We excluded subjects whose primary language was not English, subjects with education less than 12 years (to reduce educational bias), and subjects with secondary diagnoses of stroke or depression.

\section{Measures}

Language articulation and word-finding ability, hypofluency, hyperfluency, and repetition were all scored only as normal (0) or abnormal (1) in the judgment of the clinician during the conduct of the mental status examination. Animal naming was scored as the number of animals named in a minute.

The MMSE is a brief cognitive assessment measure that tests orientation, attention, concentration, recent memory, naming, repetition, comprehension, ideomotor praxis, constructional praxis, and the ability to construct a sentence. Scores range from 0 to 30 , with lower scores indicating more severe impairment. The CDR, which was designed to assess $\mathrm{AD}$ outpatients, involves a structured interview of the informant and the patient. It grades on a scale beginning with 0 (normal) and includes 0.5 (questionable dementia), 1 (mild dementia), 2 (moderate dementia), and 3 (severe dementia).

\section{Statistical Analyses}

Initial visit measures included in the analyses were demographic information (sex, race, age at initial visit, and education) and clinical measures (MMSE and CDR scores, language items from the mental status examination, and total CERAD word count). Medians and ranges are presented for the continuous measures and numbers and percentages for the dichotomous measures. For each of the measures (articulation, animal naming [number of animals named in 1 minute], fluency [word-finding difficulty in spontaneous speech, hypofluency, hyperfluency], repetition, and confrontational naming), groups were defined as normal and abnormal. Total MMSE scores for each of these groups were compared using Mann-Whitney U tests. Associations among the measures were examined using Spearman rank-order correlations. SPSS V14.0 (SPSS, Inc., Chicago, Ill.) was used to analyze the data, and statistical significance was $\mathrm{p}<.05$.

\section{Results}

A total of 486 subjects ( $66.1 \%$ women, $96.7 \%$ white) met inclusion criteria. The median age at initial visit was 74.8 years (range, 40.5-92.1) and number of years of education was 14 (range, 12-22) (Table 1). There was a significant relationship between MMSE scores and all language measures except hyperfluency (Table 2). For 3 of these measures, the number of persons with abnormal responses was less than $3 \%$ (hyperfluency $\mathrm{N}=3$, articulation $\mathrm{N}=7$, and repetition $\mathrm{N}=11$ ). Abnormal articulation did not appear in subjects with MMSE scores greater than 23 and was related to declining MMSE scores (Mann-Whitney $U, p=.0162$ ). Abnormal repetition did not appear in subjects with MMSE scores greater than 20 and was related to severity as measured by MMSE and CDR scores (Mann-Whitney U, p $=.002$ and . 0047, respectively).

Significant associations were found for the total MMSE scores with number of animals named per minute $(\rho=.59, \mathrm{df}=471, \mathrm{p}<.0001)$, CDR staging $(\rho=-0.76, \mathrm{df}=420, \mathrm{p}<$. 0001), and the CERAD word count total $(\rho=.69, \mathrm{df}=446, \mathrm{p}<.0001)$; higher scores on the 
total MMSE were related to higher scores on the number of animals named per minute and the CERAD word count total score and lower scores on CDR staging (Table 3). While education and age were significantly correlated with these 3 measures, the correlations were small $(\rho<.28)$.

\section{Conclusions}

The findings of this study are limited in that they are derived from a relatively well-educated group of white individuals presenting to a dementia clinic. However, it appears that simple measures contained in the ordinary mental status examination can be useful in detecting $\mathrm{AD}$ and in estimating its severity. We found, in agreement with the literature cited earlier, that abnormal articulation and repetition of words are unusual in $\mathrm{AD}$ (and therefore would not be useful for early detection), but when present, are associated with more advanced disease. By contrast, we found impairment in language fluency, animal naming, and confrontational naming to be common and increased in frequency with more impaired cognitive and global performance. Because animal naming is a numerical measure, changes in the number of animals named in a minute can be used as an additional measure to monitor disease progression.

Beyond the scope of this study, but equally important, is the use of language evaluation in differential diagnosis. Language evaluation is useful in differentiating other dementing illnesses from AD. For example, severe hypofluency in a person with mild memory difficulty suggests primary progressive aphasia, a symptom in the development of a frontotemporal dementia. Early onset of articulation difficulty can be a sign of many disorders affecting motor speech, including progressive supranuclear palsy and multiple sclerosis, ${ }^{16}$ and is an indication for neurologic consultation.

\section{Acknowledgments}

Supported in part by a National Institute on Aging grant numbered 2P30 AG12300.

\section{References}

1. Forbes-McKay KE, Venneri A. Detecting subtle spontaneous language decline in early Alzheimer's disease with a picture description task. Neurol Sci. 2005; 26:243-254. [PubMed: 16193251]

2. Murdoch BE, Chenery HJ, Wilks V, et al. Language disorders in dementia of the Alzheimer type. Brain Lang. 1987; 31:122-137. [PubMed: 2437993]

3. Marczinski CA, Kertesz A. Category and letter fluency in semantic dementia, primary progressive aphasia, and Alzheimer's disease. Brain Lang. 2006; 97:258-265. [PubMed: 16325251]

4. Folstein MF, Folstein SE, McHugh PR. Mini-Mental State: a practical method for grading the cognitive state of patients for the clinician. J Psychiatr Res. 1975; 12:189-198. [PubMed: 1202204]

5. Binetti G, Magni E, Cappa SF, et al. Semantic memory in Alzheimer's disease: an analysis of category fluency. J Clin Exp Neuropsychol. 1995; 17:82-89. [PubMed: 7608305]

6. Cronin-Golomb A, Keane MM, Kokodis A, et al. Category knowledge in Alzheimer's disease: normal organization and a general retrieval deficit. Psychol Aging. 1992; 7:359-366. [PubMed: 1388856]

7. Henry JD, Crawford JR, Phillips LH. Verbal fluency performance in dementia of the Alzheimer's type: a meta-analysis. Neuropsychologia. 2004; 42:1212-1222. [PubMed: 15178173]

8. Smith SR, Murdoch BE, Chenery HJ. Semantic abilities in dementia of the Alzheimer type 1: lexical semantics. Brain Lang. 1989; 36:314-324. [PubMed: 2920289]

9. Smith, SR.; Chenery, HJ.; Murdoch, BE., et al. Semantic abilities in dementia of the Alzheimer type 11: grammatical semantics. 
10. Qureshi KN, Hodkinson HM. Evaluation of a ten-question mental status test in the institutionalized elderly. Age Ageing. 1974; 3:152-157. [PubMed: 4463714] Brain Lang. 1989; 36:533-542. [PubMed: 2720369]

11. Morris JC. The Clinical Dementia Rating (CDR): current version and scoring rules. Neurology. 1993; 43:2412-2414. [PubMed: 8232972]

12. Weiner, MF. Mental status examination. In: Weiner, MF.; Lipton, AM., editors. The Dementias: Diagnosis, Treatment, and Research. Washington, DC: American Psychiatric Publishing; 2003. p. 487-494.

13. Morris JC, Heyman A, Mohs RC, et al. The Consortium to Establish a Registry for Alzheimer's Disease (CERAD), pt 1: clinical and neuropsychological assessment of Alzheimer's disease. Neurology. 1989; 39:1159-1165. [PubMed: 2771064]

14. McKhann G, Drachman D, Folstein M, et al. Clinical diagnosis of Alzheimer's disease: report of the NINCDS-ADRDA work group under the auspices of the Department of Health and Human Services Task Force on Alzheimer's Disease. Neurology. 1984; 34:939-944. [PubMed: 6610841]

15. Ranginwala NA, Weiner MF, Hynan LS, et al. Clinical criteria for the diagnosis of Alzheimer's disease: still good after all these years. Am J Geriatr Psychiatry. In press.

16. Lipton, AM.; Weiner, MF. Differential diagnosis. In: Weiner, MF.; Lipton, AM., editors. The Dementias: Diagnosis, Treatment, and Research. Washington, DC: American Psychiatric Publishing; 2003. p. 137-180. 


\section{Take-Home Points}

- Animal naming (semantic or category fluency) is a simple measure that can be used to follow the progression of Alzheimer's disease.

- The mechanics of language are well preserved in patients with early Alzheimer's disease.

- Education and age should be considered in evaluation of language in patients with Alzheimer's disease. 
Table 1

Summary Information for Continuous Measures Among Subjects With Alzheimer's Disease

\begin{tabular}{lrr}
\hline Statistic & Median & Range \\
\hline${\text { Education, } \mathrm{y}^{a}}$ & 14 & $12-22$ \\
Age at initial visit, $\mathrm{y}^{a}$ & 74.8 & $40.5-92.1$ \\
CERAD word count total $^{b}$ & 10 & $0-33$ \\
No. of animals named in 1 min $^{c}$ & 8 & $0-32$ \\
MMSE score $^{a}$ & 21 & $0-30$ \\
CDR score $^{d}$ & 1 & $0.5-3$ \\
\hline
\end{tabular}

$a_{\mathrm{N}}=486$.

${ }^{b} \mathrm{~N}=448$.

$c_{\mathrm{N}}=473$

$d_{\mathrm{N}}=422$.

Abbreviations: $\mathrm{CDR}=$ Clinical Dementia Rating scale, $\mathrm{CERAD}=$ Consortium to Establish a Registry for Alzheimer's Disease, $\mathrm{MMSE}=\mathrm{Mini}-$ Mental State Examination. 
:

:

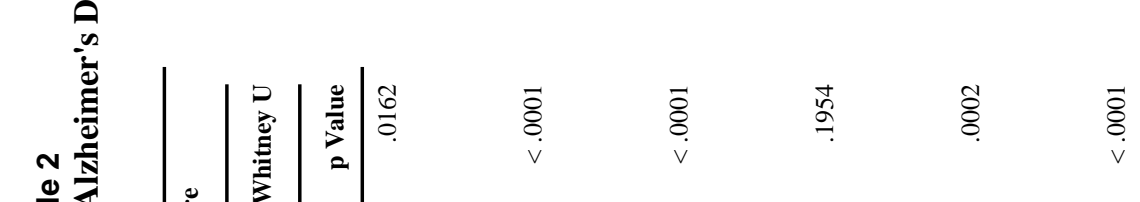


